\title{
Value of dual testing for identifying tuberculous infection ${ }^{\text {is }}$
}

\author{
S. Radhakrishna, T.R. Frieden, R. Subramani, P.R. Narayanan*
}

Tuberculosis Research Centre (ICMR), Mayor V.R. Ramanathan Road, Chetput, Chennai 600031 , India

Received 13 June 2005; received in revised form 13 June 2005; accepted 24 June 2005

\author{
KEYWORDS \\ Dual testing; \\ PPD-S; \\ PPD-B; \\ Identifying infected
}

\begin{abstract}
Summary
Setting: A rural community in Chingleput district in Tamil Nadu state in south India. Objective: To determine the value of dual testing with PPD-S and PPD-B for identifying subjects with a tuberculous infection.

Design: About 240,000 subjects in rural south India, all of whom were tested initially with PPD-S and PPD-B, were followed up for 15 years, mainly by total population survey once in every $2 \frac{1}{2}$ years. The incidence of culture-positive tuberculosis was estimated using life-table technique.

Results: Among 17,530 subjects with an intermediate reaction $(8-11 \mathrm{~mm})$ to PPD-S at intake, 285 with an induration to PPD-S exceeding the induration to PPD-B by at least $2 \mathrm{~mm}$, had a significantly higher incidence of culture-positive tuberculosis than the remaining (154 and 93 per 100,000), and similarly 481 who had an induration of $<10 \mathrm{~mm}$ to PPD-B compared to those with $\geqslant 10 \mathrm{~mm}(131$ and 93 per 100,000). These subjects may be regarded as having a tuberculous infection.

Infection with non-tuberculous mycobacteria conferred protection of about $30 \%$ against the development of tuberculosis over a 15-year period.

Conclusion: In subjects with an intermediate reaction $(8-11 \mathrm{~mm})$ to PPD-S, dual testing with PPD-B enabled identification of those with a tuberculous infection. Most of the reactions were due to non-tuberculous mycobacteria.

(c) 2005 Elsevier Ltd. All rights reserved.
\end{abstract}

\footnotetext{
This report was prepared by Dr. S. Radhakrishna, former Director, Institute for Research in Medical Statistics, Madras Chapter (ICMR) who had the primary responsibility for analysis and writing of this manuscript. Dr. Thomas R. Frieden, Medical Officer (TB), WHO-SEARO, participated in design of the analysis and writing of the manuscript. Mr. R. Subramani, Assistant Director, Tuberculosis Research Centre, Chennai was responsible for data management and data output.

*Corresponding author. Tel.: +914428362525; fax: +914428362528 .

E-mail address: nrparanj@md2.vsnl.net.in (P.R. Narayanan).
}

\section{Introduction}

In an earlier publication from this Centre, based on a 15-year follow-up of a large rural population in south India, we had demonstrated the presence of an association between sensitivity to PPD-S at intake and the incidence of culture-positive tuberculosis, which persisted after allowing for the effect of sex and age. ${ }^{1}$ While the incidence in 
'infected' subjects $(\geqslant 12 \mathrm{~mm})$ was substantially higher than that in the 'uninfected' $(0-7 \mathrm{~mm})$, the incidence in those with $8-11 \mathrm{~mm}$ was numerically higher than in the 'uninfected' but not significantly so; further, it was significantly lower than that in infected subjects. These findings suggest that the 8-11 mm group might be a mixed group, consisting largely of uninfected subjects. Dual testing with PPD-B has been reported to be useful for classifying such subjects as uninfected or infected. ${ }^{2,3}$ We present here the findings from our large and unique data set, employing a multivariate analytical tool. Two approaches were investigated, one based on the excess of PPD-S induration over PPD-B induration, and the other based on a dichotomy of the absolute value of the PPD-B induration.

\section{Materials and methods}

A double-blind randomized controlled trial was initiated in 1968 in about 240,000 subjects in rural south India to assess the protective efficacy of BCG vaccination. In this trial, all individuals aged one year and above were tested with three international units of PPD-S and 10 units of PPD-B, derived from Mycobacterium intracellulare. (PPD-S was supplied from the Antigen production laboratory, Atlanta, Georgia, during the first six months of the intake, and from the BCG department of Statens Seruminstitut, Copenhagen for the next two years. PPD-B was from the Antigen production laboratory in Atlanta, Georgia.) The participants were subsequently allocated at random to a placebo, a low dose of BCG $(0.01 \mathrm{mg})$ or a high dose of BCG $(0.1 \mathrm{mg})$. Subjects who had a positive smear/ culture or BCG scar at intake, or were not assessed for scar status or not 'vaccinated' in the trial were excluded. Of the remaining, only those with a normal radiograph at intake were considered for determining the incidence of tuberculosis. The initial study population was 237,276 (Placebo 79,526, BCG low dose 78,981, BCG high dose $78,769)$. Since there were no differences in incidence between the three series, ${ }^{4,5}$ the total population of 237,276 was considered for all further analyses.

\section{Investigations}

Radiographic examination, followed by sputum examination of those with an abnormal radiograph, was undertaken to detect all cases of tuberculosis over the next 15 years. The detection was through total population survey once in $2 \frac{1}{2}$ years, selective follow-up of subjects at higher risk once in 10 months, and passive surveillance through a specially established clinic in the area. These procedures were for subjects with an induration of $0-15 \mathrm{~mm}$ to PPD-S at intake. In those with an induration of $>15 \mathrm{~mm}$, from $7 \frac{1}{2}$ years onwards follow-up was restricted to subjects at especially high risk (e.g. suspect shadows on radiography) and a random sample of one-third of the others. Full details have been reported earlier. ${ }^{4,5}$

\section{Definition of tuberculous infection based on induration to PPD-S}

Considering the distribution of PPD-S results in 68,415 children aged 0-9 years, there was no clear separation between the presumably uninfected and the presumably infected subjects, but the dip between the two distributions was approximately at $12 \mathrm{~mm} .{ }^{4}$ Further, the lower $95 \%$ confidence limit for 556 patients with culture-positive tuberculosis was $12.6 \mathrm{~mm} .{ }^{1}$ Therefore, subjects with an induration of $\geqslant 12 \mathrm{~mm}$ were regarded as very likely to have had a tuberculous infection ('infected'). The others were divided in to two groups, i.e. $0-7 \mathrm{~mm}$, comprising subjects who were very unlikely to have had a tuberculous infection ('uninfected'), the rest $(8-11 \mathrm{~mm})$ being an intermediate group.

\section{Definition of non-tuberculous infection based on induration to PPD-B}

Considering the distribution of PPD-B results in the same 68,415 children, there was no clear division between non-reactors and reactors, but the lowest frequency was at $11 \mathrm{~mm}$. Further, in 62,290 adults aged $>35$ years, most of whom are expected to be reactors, $98 \%$ had an induration of $>10 \mathrm{~mm}$, which was therefore taken as the cut-off for classifying a subject as having a non-tuberculous mycobacterial infection. The same definition had been employed in our earlier reports also. ${ }^{4,5}$

\section{Assessment of additional value of PPD-B sensitivity}

To explore the additional value of PPD-B sensitivity, two approaches were employed, each resulting in six subgroups. In the first, the induration to PPD-S $(0-7,8-11,>12 \mathrm{~mm})$ was considered with the difference in induration to PPD-S (S) and PPD-B (B) 
(less than $2 \mathrm{~mm}, 2 \mathrm{~mm}$ or more); three groups were initially considered for those with PPD-S induration of $0-7$ and $8-11 \mathrm{~mm}$, as suggested by Edwards et al., ${ }^{3}$ namely, difference $(\mathrm{S}-\mathrm{B})$ of -2 or less; difference of $-1,0,1$; difference of 2 or more, but the first two were combined as they were found to have very similar risks. In the second approach, the induration to PPD-S was considered along with a dichotomous classification of the induration to PPDB $(10 \mathrm{~mm}$ or more, $0-9 \mathrm{~mm})$.

\section{Estimation of incidence}

For each period of $2 \frac{1}{2}$ years, the population at risk was stratified by sex, age and tuberculin age and tuberculin sensitivity at intake, and the death rate and the incidence of tuberculosis were estimated for each subgroup. Using these in a life table analysis, the numbers of culture-positive cases (irrespective of smear) were determined per 100,000 persons in each sub-group. Assuming that the absentees in every subgroup had similar outcomes as those investigated, and employing suitable weighting procedures, the incidence (per 100,000 ) of culture-positive tuberculosis was computed for each of the tuberculin sensitivity groups set out above. This procedure was employed to allow for differential losses in the follow-up that have been described in detail in the earlier communication. ${ }^{1}$

\section{Statistical methods}

Since the period of follow-up was not uniform for all subjects (planned duration varied from 7.5 to 15 years, and there were interim losses due to migration etc. ${ }^{4,5}$ ), Cox's proportional hazard model $^{6}$ was employed to assess the statistical significance of contrasts between various tuberculin sensitivity groups, both for the univariate case and for the multivariate situation when other prognostic factors such as age at intake, sex and vaccination status were also taken into account.

\section{Results}

\section{Role of PPD-B sensitivity in identifying those with tuberculous infection}

The annual incidence of culture-positive tuberculosis in those with PPD-S $=0-7 \mathrm{~mm}$ was unrelated to the magnitude of the difference in sensitivity between PPD-S and PPD-B, i.e. 57 and 53 per $100,000(p=0.58)$ in those with difference (S-B) of $\geqslant 2$ and $<2 \mathrm{~mm}$, respectively (Table 1 ). In those with PPD-S $=8-11 \mathrm{~mm}$, however, the corresponding incidences were 154 and 93, respectively, suggesting that the former category (PPD-S reaction larger than PPD- $B$ reaction by at least $2 \mathrm{~mm}$ ) had a higher risk $(p=0.28)$ due probably to a tuberculous infection; this contrast was more marked in adults aged 15 years or more, the incidences being 304 and 116 , respectively $(p<0.01)$. In subjects with a definitive tuberculous infection $(S \geqslant 12 \mathrm{~mm})$, the corresponding incidences were 355 and 301, respectively $(p<0.001)$. Subjects with an PPD-S excess of $2 \mathrm{~mm}$ or more were younger than those with an excess of less than $2 \mathrm{~mm}$ (Table $1, p<0.01$ ). Also, the proportion of males differed significantly between the two groups (Table $1, p<0.01$ ) in subjects with $0-7 \mathrm{~mm}(49.2 \%, 46.3 \%)$ and $\geqslant 12 \mathrm{~mm}$ $(48.0 \%, 56.6 \%)$. As age and sex are both associated with tuberculosis incidence, ${ }^{1}$ we undertook a

Table 1 Incidence of culture-positive tuberculosis, age at intake, sex and Adjusted risk of tuberculosis in various groups based on induration to PPD-S and excess induration of PPD-S over PPD-B.

\begin{tabular}{|c|c|c|c|c|c|c|c|c|c|c|c|}
\hline \multirow[t]{2}{*}{$\begin{array}{l}\text { Induration to } \\
\text { PPD-S (mm) }\end{array}$} & \multirow[t]{2}{*}{$\begin{array}{l}\text { Excess of } \\
S \text { over B (S-B) }\end{array}$} & \multirow[t]{2}{*}{$\begin{array}{l}\text { Number } \\
\text { at risk }\end{array}$} & \multirow[t]{2}{*}{$\begin{array}{l}\text { Annual } \\
\text { incidence* }\end{array}$} & \multicolumn{6}{|c|}{$\begin{array}{l}\text { Percentage of subjects } \\
\text { by age (years) and by sex }\end{array}$} & \multirow[t]{2}{*}{$\begin{array}{l}\text { Adjusted } \\
\mathrm{RR}^{\dagger}\end{array}$} & \multirow[t]{2}{*}{$p$} \\
\hline & & & & 0-14 & $15-24$ & $25-44$ & $\geqslant 45$ & Male & Female & & \\
\hline \multirow[t]{2}{*}{$0-7$} & $\mathrm{~nm}$ & 106728 & 53 & 72.7 & 12.4 & 10.4 & 4.5 & 46.3 & 53.7 & 1.00 & \\
\hline & $2 \mathrm{~mm}$ or more & 1794 & 57 & 94.0 & 1.7 & 2.1 & 2.2 & 49.2 & 50.8 & 1.45 & 0.34 \\
\hline \multirow[t]{2}{*}{$8-11$} & Less than $2 \mathrm{~mm}$ & 17245 & 93 & 33.2 & 21.5 & 28.4 & 16.9 & 46.3 & 53.7 & 1.00 & \\
\hline & $2 \mathrm{~mm}$ or more & 285 & 154 & 60.1 & 10.5 & 10.5 & 18.9 & 47.7 & 52.3 & 3.23 & 0.02 \\
\hline \multirow[t]{2}{*}{$\geqslant 12$} & Less than $2 \mathrm{~mm}$ & 71090 & 301 & 13.0 & 18.5 & 43.0 & 25.4 & 56.6 & 43.4 & 1.00 & \\
\hline & $2 \mathrm{~mm}$ or more & 40134 & 355 & 19.7 & 18.6 & 38.9 & 22.8 & 48.0 & 52.0 & 1.42 & $<0.001$ \\
\hline
\end{tabular}

\footnotetext{
*Annual incidence of culture-positive tuberculosis (per 100 000), smear-positive or smear-negative, over 15 years.

${ }^{\dagger}$ Adjusted risk of tuberculosis after allowing for age at intake, sex and vaccination status.
} 
multivariate analysis within each of these 3 groups $(0-7,8-11, \geqslant 12 \mathrm{~mm})$. This showed that subjects whose PPD-S induration exceeded PPD-B induration by at least $2 \mathrm{~mm}$ consistently had a higher adjusted relative risk (over 15 years) than those with an excess of less than $2 \mathrm{~mm}$, the contrast being statistically significant in subjects with $8-11 \mathrm{~mm}$ (Adj. RR $=3.2, p=0.02$ ) and $\geqslant 12 \mathrm{~mm}$ (Adj. RR $=1.4, p<0.001)$; in subjects with $0-7 \mathrm{~mm}$, the adjusted risk was $1.4(p=0.34)$.

A multivariate analysis on the total data set yielded two important findings (Table 3, left-hand side). One is that the adjusted RR for subjects with $S=8-11 \mathrm{~mm}$ in whom the excess of $S$ over $B$ was less than $2 \mathrm{~mm}$, was $1.04(p=0.66)$, i.e. not significantly different from that in subjects with $S=0-7 \mathrm{~mm}$. The other is that subjects with $S=$ $8-11 \mathrm{~mm}$ and an excess of at least $2 \mathrm{~mm}$ had an adjusted RR of 2.8 that was significantly higher than that in subjects with $S=0-7 \mathrm{~mm}(p=0.04)$, and the same as that in subjects with a tuberculous infection $(S \geqslant 12 \mathrm{~mm})$. Similar patterns were evident with 5- and 10-year follow-up periods.
An alternative approach is to sub-divide individuals with $8-11 \mathrm{~mm}$ to PPD-S into two groups according to their PPD-B reactor status (Table 2). The group with an induration of $\geqslant 10 \mathrm{~mm}$ to PPD-B, suggesting the presence of a non-tuberculous mycobacterial infection (with or without a concomitant tuberculous infection), had a culturepositive incidence of 93 per 100,000 , which was lower than the corresponding incidence of 131 per 100,000 in those with $<10 \mathrm{~mm} \quad(p=0.31)$. In subjects with $0-7 \mathrm{~mm}$ initially to PPD-S, the difference was in the opposite direction (63 and 34 ), while in those with $\geqslant 12 \mathrm{~mm}$, the difference was relatively small (316 and 323) and nonsignificant.

Subjects with an induration to PPD-B of $\geqslant 10 \mathrm{~mm}$ were older than those with an induration of $<10 \mathrm{~mm}$ (Table 2, $p<0.001$ ). Also, the proportion of males differed significantly between the two PPD-B groups in subjects with PPD-S $=0-7 \mathrm{~mm}$ $(44.7 \%, 49.2 \%)$ and in those with $\geqslant 12 \mathrm{~mm}(53.6 \%$, 45.9\%). Multivariate analysis within each of these three groups showed that the adjusted relative risk

Table 2 Incidence of culture-positive tuberculosis, age at intake, sex and Adjusted risk of tuberculosis in various groups based on indurations to PPD-S and PPD-B.

\begin{tabular}{|c|c|c|c|c|c|c|c|c|c|c|c|}
\hline \multirow[t]{2}{*}{$\begin{array}{l}\text { Induration to } \\
\text { PPD-S (mm) }\end{array}$} & \multirow[t]{2}{*}{$\begin{array}{l}\text { Induration to } \\
\text { PPD-B (mm) }\end{array}$} & \multirow[t]{2}{*}{$\begin{array}{l}\text { Number } \\
\text { at risk }\end{array}$} & \multirow[t]{2}{*}{$\begin{array}{l}\text { Annual } \\
\text { incidence* }\end{array}$} & \multicolumn{6}{|c|}{$\begin{array}{l}\text { Percentage of subjects } \\
\text { by age (years) and by sex }\end{array}$} & \multirow[t]{2}{*}{$\begin{array}{l}\text { Adjusted } \\
\mathrm{RR}^{\dagger}\end{array}$} & \multirow[t]{2}{*}{$p$} \\
\hline & & & & $0-14$ & $15-24$ & $25-44$ & $\geqslant 45$ & Male & Female & & \\
\hline \multirow[t]{2}{*}{$0-7$} & $\geqslant 10$ & 69,530 & 63 & 61.0 & 17.7 & 15.1 & 6.3 & 44.7 & 55.3 & 1.00 & \\
\hline & $0-9$ & 38,992 & 34 & 94.5 & 2.5 & 1.7 & 1.3 & 49.2 & 50.8 & 1.12 & 0.36 \\
\hline \multirow[t]{2}{*}{$8-11$} & $\geqslant 10$ & 17,049 & 93 & 32.8 & 21.7 & 28.7 & 16.9 & 46.3 & 53.7 & 1.00 & \\
\hline & $0-9$ & 481 & 131 & 62.6 & 10.0 & 9.6 & 17.9 & 46.0 & 54.0 & 3.23 & $<0.01$ \\
\hline \multirow[t]{2}{*}{$\geqslant 12$} & $\geqslant 10$ & 109,919 & 316 & 15.2 & 18.6 & 41.8 & 24.5 & 53.6 & 46.4 & 1.00 & \\
\hline & $0-9$ & 1305 & 323 & 39.3 & 13.7 & 23.1 & 23.9 & 45.9 & 54.1 & 1.49 & 0.03 \\
\hline
\end{tabular}

*Annual incidence of culture-positive tuberculosis (per 100 000), smear-positive or smear-negative, over 15 years.

${ }^{\dagger}$ Adjusted risk of tuberculosis after allowing for age at intake, sex and vaccination status.

Table 3 Adjusted relative risk of tuberculosis (15-year follow-up) by PPD-S and PPD-B induration at intake.

\begin{tabular}{|c|c|c|c|c|c|c|c|c|c|c|}
\hline $\begin{array}{l}\text { Induration } \\
\text { to PPD-S } \\
(\mathrm{mm})\end{array}$ & $\begin{array}{l}\text { Excess of } \\
\text { PPDS over } \\
\text { PPD-B } \\
(\mathrm{mm})\end{array}$ & $\begin{array}{l}\text { Number } \\
\text { at risk }\end{array}$ & $\begin{array}{l}\text { Annual } \\
\text { incidence* }\end{array}$ & $\begin{array}{l}\text { Adjusted } \\
\mathrm{RR}^{\dagger}\end{array}$ & $P$ & $\begin{array}{l}\text { Induration } \\
\text { to PPD-B } \\
(\mathrm{mm})\end{array}$ & $\begin{array}{l}\text { Number } \\
\text { at risk }\end{array}$ & $\begin{array}{l}\text { Annual } \\
\text { incidence* }\end{array}$ & $\begin{array}{l}\text { Adjusted } \\
\mathrm{RR}^{\dagger}\end{array}$ & $p$ \\
\hline $0-7$ & - & 108,522 & 52 & 1.00 & & - & 108,522 & 52 & 1.00 & \\
\hline \multirow[t]{2}{*}{$8-11$} & $<2$ & 17,245 & 93 & 1.04 & 0.66 & $\geqslant 10$ & 17,049 & 93 & 1.03 & 0.73 \\
\hline & $\geqslant 2$ & 285 & 154 & 2.79 & 0.04 & $0-9$ & 481 & 131 & 2.68 & 0.02 \\
\hline$\geqslant 12$ & - & 111,224 & 332 & 2.78 & $<0.001$ & - & 111,224 & 332 & 2.78 & $<0.001$ \\
\hline
\end{tabular}

*Annual incidence of culture-positive tuberculosis (per 100,000), smear-positive or smear-negative, over 15 years.

${ }^{\dagger}$ Adjusted risk of tuberculosis after allowing for age at intake, sex and vaccination status. 
was consistently higher in subjects with an induration of $<10 \mathrm{~mm}$ to PPD-B, the contrast being statistically significant in subjects with $S=$ $8-11 \mathrm{~mm}$ (Adj. RR $=3.2, p<0.01$ ) and in those with $S \geqslant 12 \mathrm{~mm}$ (Adj. $R R=1.5, p=0.03$ ); in those with $0-7 \mathrm{~mm}$, the adjusted risk was $1.1(p=0.36)$.

Multivariate analysis of the entire data set showed that the adjusted RR for $S=8-11, B \geqslant 10$ was 1.03 , i.e. not significantly different from that in subjects with $S=0-7 \mathrm{~mm}$ (Table 3 , right-hand side). Subjects with $S=8-11, B<10$ had a significantly higher adjusted RR of $2.7(p=0.02)$, that was very similar to that in subjects with a tuberculous infection (2.8).

\section{Effect of non-tuberculous mycobacterial infection on incidence}

Subjects with a non-tuberculous mycobacterial infection $(B \geqslant 10 \mathrm{~mm})$ had a lower adjusted relative risk than those without such an infection. In subjects with $S=0-7 \mathrm{~mm}$, the adjusted RR was 0.89 (reciprocal of 1.12 in Table 2), which is suggestive but not statistically significant $(p=0.36)$. It was 0.31 (reciprocal of 3.23 in Table 2) for subjects with $S=8-11 \mathrm{~mm}(p<0.01)$, and 0.67 (reciprocal of 1.49 in Table 2) for those with $S \geqslant 12 \mathrm{~mm}(p=0.03)$. For the total population, the adjusted RR (weighted estimate of the three separate estimates) was approximately 0.69 , indicating an overall protective efficacy of $31 \%$.

\section{Discussion}

The classification of individuals as infected (with tubercle bacilli) or uninfected has important clinical and programme implications. For instance, it could form the basis for BCG vaccination in the uninfected or chemoprophylaxis to the infected, or the computation of the annual risk of tuberculous (ARTI) infection, an important index for the programme manager. A frequently employed method is to choose the cut-off point as the dip (antimode) in the distribution of PPD-S induration in relatively young subjects-for example, children aged less than 10 years. With this method, definite non-reactors and definite reactors are identified, but there is an element of uncertainty about some due to subjectivity in determining the cut-off point. In a preliminary report on 624,860 white navy recruits in the USA, Palmer and Edwards ${ }^{2}$ concluded that the use of PPD-B in addition to PPD$S$ in such situations could significantly improve the efficiency of classification, as they found striking differences in morbidity between subjects whose induration to PPD-S exceeded that to PPD-B by at least 2,1 to -1 , or $-2 \mathrm{~mm}$ or less. The larger study in $1,124,863$ recruits confirmed this conclusion, ${ }^{3}$ the incidences being 272, 127 and 29 per 100,000, respectively, with the incidence in the last group being similar to that in non-reactors (36 per 100,000).

In contrast, Raj Narain and colleagues ${ }^{7}$ concluded that dual testing with PPD-B is unlikely to serve a useful purpose in south India. Their conclusion was not based on the risk of developing tuberculosis (such information was not available at the time), but the finding that the PPD-B reaction was larger than the PPD-S reaction (which was at least $12 \mathrm{~mm}$ ) in as many as $25 \%$ of 1626 patients with culturepositive tuberculosis, and $9.4 \%$ of 106 children aged less than 5 years. The corresponding proportions in our study were $28 \%$ in 627 culture-positive patients and $27 \%$ in 1360 children aged less than 5 years. From Canada, Menzies et al. reported that sensitivity to PPD-B had an important effect on lowgrade reactions (range of $5-9 \mathrm{~mm}$ ) to PPD-T (bio equivalent of PPD-S), through cross-reactivity, but negligible effect on reactions of $10+\mathrm{mm}^{8}$

In subjects with an intermediate reaction $(8-11 \mathrm{~mm})$ to PPD-S, our findings agree with those in navy recruits in the USA. ${ }^{2,3}$ After allowing for the effect of associated factors such as age and sex, the incidence in 285 subjects with whose $S$ induration exceeded $B$ induration by $2 \mathrm{~mm}$ or more was significantly higher than that in subjects with a difference of less than $2 \mathrm{~mm}$, and similar to that in subjects with a tuberculous infection. Similarly, there were 481 subjects (including all of the 285 above) who were not reactors to PPD-B (0-9 mm) and had an incidence similar to that in infected subjects. In all, therefore, 481 (2.7\%) of the 17,530 subjects could be identified as probably infected, suggesting that subjects with an intermediate size reaction to PPD-S $(8-11 \mathrm{~mm})$ constitute a mixed group of uninfected and infected subjects, with the vast majority $(97 \%)$ not having a tuberculous infection. Further, even among subjects with a definite tuberculous infection (PPD- $S \geqslant 12 \mathrm{~mm}$ ), a large subset could be identified (PPD- $B \geqslant 10 \mathrm{~mm}$ ) that had a $30 \%$ lower risk $(p=0.03)$. Thus, dual testing with PPD-B proved to be useful in this community, but its routine use in real life situations has to take into account the labour and cost involved, besides ensuring availability of the PPD$B$ antigen, the production of which has been discontinued in the USA and in Statens SerumInstitut, Copenhagen. Finally, the utility of this supplemental test with PPD-B in distinguishing tuberculosis infection from non-specific reactivity 
at the individual level suggests that something closer than what is currently available to a definitive diagnosis of tuberculosis infection is an achievable goal.

The focus of the present investigation has been on the classification of individual subjects as infected or uninfected for clinical purposes (e.g. BCG vaccination, chemoprophylaxis). For the epidemiologist or programme manager, who would probably be satisfied with an estimate of the proportion infected, 'mixture' models are an attractive alternative, especially in situations where there is a high prevalence of environmental mycobacteria. ${ }^{9-12}$ In this approach, the tuberculin test results are assumed to be a mixture of three 'Normal' distributions, one for reactors due to a tuberculous infection, one for reactors due to cross infection with environmental mycobacteria, and the third for non-reactors (i.e. neither infected with tuberculous nor non-tuberculous mycobacteria); the proportions in each category, together with the means and standard deviations of the three Normal distributions, are then estimated by maximum likelihood analysis. The ARTI computed from a Mixture model in Karnataka, ${ }^{9}$ was almost the same as that obtained by the conventional method of a critical cut-off, but in Afghanistan the former was substantially smaller. ${ }^{10} \mathrm{~A}$ study in Korea showed that the approach is also promising in the context of changing tuberculin reaction sizes over time, ${ }^{11}$ while a more theoretical study concluded that the method is an useful adjuvant when the diagnostic test lacks in specificity as a result of cross reactions. ${ }^{12}$

Palmer and Long cited laboratory studies and human studies to suggest that infections with mycobacteria other than BCG create some capacity to modify the course of subsequent virulent disease, and concluded that infections with such organisms constitute a kind of 'natural' vaccination that resembles vaccination with BCG. ${ }^{13}$ The magnitude of protection from non-tuberculous mycobacteria (based on univariate analysis) was $60 \%$ over a 10-year period in the United Kingdom, ${ }^{14}$ and $58 \%$ over 3 years in south India. ${ }^{15}$ Our estimate, based on multivariate analysis that allows for the effect of age and sex, is about $30 \%$ over a 15 -year period. These findings suggest that infection with PPD-B prevented progression to tuberculosis disease in those with an initial tuberculous infection. Evidence of cross protection from natural exposure to environmental bacteria has also been suggested from Malawi, ${ }^{16}$ where the lowest rates of tuberculosis were found in subjects with low-grade sensitivity to PPD-S. ${ }^{17}$

\section{Acknowledgements}

The authors are grateful to Professor N.K. Ganguly, the Director General of the Indian Council of Medical Research for his constant encouragement. The authors are also grateful to Dr. P.R. Narayanan, Director of Tuberculosis Research Centre for his guidance and invaluable support. The authors acknowledge the role of the former Directors of the Tuberculosis Research Centre Dr. S.P. Tripathy and Dr. R. Prabhakar, and the Project Directors of the Tuberculosis Prevention Trial (late) Dr. Raj Narain and Dr. G.V.J. Baily, in the conduct of the Tuberculosis Prevention Trial. The authors also gratefully acknowledge the invaluable contributions of the entire scientific, technical, field and administrative staff (retired and currently in service) of the Tuberculosis Prevention Trial and the Tuberculosis Research Centre involved in the trial, notably Mr. R.S. Vallishayee, Dr. S. Mayurnath, Dr. Manjula Datta, Mrs. M.P. Radhamani, Mr. A.M. Diwakara, and (late) Dr. J. Guld, Consultant, World Health Organization. The authors gratefully acknowledge numerous suggestions regarding analyses and advice about presentation made by Prof. G.W. Comstock, Johns Hopkins University, Baltimore, USA.

The analysis and presentation of this data was supported in part by World Health Organization with financial assistance provided by the United States Agency for International Development under the Model DOTS Project.

\section{References}

1. Tuberculosis Research Centre, Chennai. Association of initial tuberculin sensitivity, age and sex with the incidence of tuberculosis in south India: a 15-year follow-up. Int J Tuberc Lung Dis 2003;7:1-9.

2. Palmer CE, Edwards LB. Identifying the tuberculous infected: the dual test technique. JAMA 1968;205:167-9.

3. Edwards LB, Acquaviva FA, Livesay VT. Identification of tuberculous infected. Am Rev Respir Dis 1973;108: 1334-9.

4. Tuberculosis Prevention Trial, Madras. Trial of BCG vaccines in south India for tuberculosis prevention. Ind $\mathrm{J}$ Med Res 1980;72(Suppl):1-74.

5. Tuberculosis Research Centre, Chennai. Fifteen-year followup of BCG vaccines in south India for tuberculosis prevention. Ind J Med Res 1999;110:56-69.

6. Cox DR. Regression models and life tables (with discussion). $J$ R Stat Soc B 1972;34:187-220.

7. Raj Narain, Vallishayee RS, Reddy AV. Value of dual testing with PPD-S and PPD-B. Ind J Med Res 1978;68:204-19.

8. Menzies D, Chun Ho Chan, Vissandjee B. Impact of immigration on tuberculosis infection among Canadian-born schoolchildren and young adults in Montreal. Am J Respir Crit Care Med 1997;156(6):1915-21. 
9. Savanur SJ, Chadha VK, Jagannatha PS. Mixture model for analysis of tuberculin surveys. Indian J Tuberc 2002;49:147-51.

10. Dubuis M, Fiekert K, Neuenschwander BE, Rieder HL. A tuberculin skin test survey among Afghan children in Kabul. Int J Tuberc Lung Dis 2004;8:1065-72.

11. Neuenschwander BE, Zwalhen M, Kim SJ, Engel RR, Rieder $\mathrm{HL}$. Trends in the prevalence of infection with Mycobacterium tuberculosis in Korea from 1965 to 1995; an analysis of seven surveys by mixture models. Int $J$ Tuberc Lung Dis 2000;4:719-29.

12. Nagelkerke NJD, Borgdorff MW, Kim SJ. Logistic discrimination of mixtures of $M$. tuberculosis and nonspecific tuberculin reactions. Statist Med 2001;20:1113-24.

13. Palmer CE, Long MW. Effects of infection with atypical mycobacteria on BCG vaccination and tuberculosis. Am Rev Respir Dis 1966;94:553-68.
14. British Medical Research Council BCG and vole bacillus vaccines in the prevention of tuberculosis in adolescence and early adult life. Third report. $\mathrm{Br}$ Med $\mathrm{J}$ 1963;1:973-8.

15. Raj Narain, Naganna K, Pyare Lal. Nonspecific sensitivity and its influence on incidence of pulmonary tuberculosis. Am Rev Respir Dis 1972;105:578-85.

16. Fine PEM, Floyd S, Stanford JL, Nkhosa P, Kasunga A, Chaguluka S, Warndorff DK, Jenkins PA, Yates M, Ponnighaus JM. Environmental mycobacteria in northern Malawi: implications for the epidemiology of tuberculosis and leprosy. Epidemiol Infect 2001;126:379-87.

17. Fine PEM, Sterne JAC, Ponnighaus JM, Rees RJW. Delayedtype hypersensitivity, mycobacterial vaccines and protective immunity. Lancet 1994;344:1245-9.

Available online at www.sciencedirect.com

science $\boldsymbol{d}$ Directo 